\title{
Looking for a chronic care model in COPD patients
}

\author{
Enrico Clini D, Ivana Castaniere and Roberto Tonelli
}

Affiliation: Dept of Medical and Surgical Sciences, University of Modena Reggio Emilia and University Hospital of Modena-Policlinico, Modena, Italy.

Correspondence: Enrico Clini, Dept of Medical and Surgical Sciences, University of Modena, University Hospital of Modena, Via del Pozzo 71, Modena 41141, Italy. E-mail: enrico.clinidunimore.it

@ERSpublications

A chronic care model in primary care is feasible and effective for the COPD population even at an early stage http://ow.ly/yZzR30gE26r

Cite this article as: Clini E, Castaniere I, Tonelli R. Looking for a chronic care model in COPD patients. Eur Respir J 2018; 51: 1702087 [https://doi.org/10.1183/13993003.02087-2017].

Chronic conditions demand "chronic" interventions intended for their holistic and thorough management, which is definitely embraced in the so-called chronic care model (CCM) [1]. In chronic obstructive pulmonary disease (COPD), an increasing body of evidence stresses the need for this patient-focused approach to care [2]. Since its first application, the chronic care method has needed to identify the ideal subset of patients that would largely benefit from its practice in real life and the most impactful areas of intervention. Recovery from hospitalisation following admission due to acute exacerbations is often recognised as the ideal time during which to educate the patient on how to react to health deterioration. Indeed, the transition from hospital back to the community represents a critical process, failure of which is associated with higher rates of rehospitalisation [3].

Although this is undoubtedly true, choosing a specific subset of COPD patients might act as a selection bias limiting the potential benefits to a specific phase along the course of the disease. Fromer [4] thus suggested a proactive strategy to reduce the burden of COPD in the early stages, requiring a collaborative strategy in the primary care setting. COPD self-management programmes have thus far been demonstrated to improve patients' quality of life and healthcare use in secondary care settings [5], which do not preclude its applicability even to general practice. Self-management programmes must be focused on the needs of each particular patient and ought to be tailored to a comprehensive dimension that only the primary care setting can actually manage. Moreover, routine monitoring of clinical outcomes and health status should become a shared responsibility between healthcare professionals and well trained patients [6].

In terms of methodology, recent evidence shows how self-management education should not be limited to simple informative activities and should be structured as a coaching approach aimed at empowering patients to modify their behaviours [7].

The CCM is based on the assumption that effective behavioural changes can only be achieved through the empowerment of patients' self-efficacy. Patients who develop enough confidence in their capacity to positively react to specific events are more prone to change and to maintain this attitude. Adequate self-management of health outcomes is defined as increased self-efficacy, individual mastery and effective integration of self-management skills on a day-to-day basis [2]. These changes in resilience should ultimately result in improved clinical outcomes and reduced healthcare costs [5].

Received: Oct 102017 | Accepted: Oct 132017

Conflict of interest: None declared

Copyright @ERS 2018 
The study by STEURER-STEY et al. [8] expanded the knowledge from other published experiences of strategies to improve self-management interventions and change behaviours in a primary care setting [4, 7]. In particular, by comparing two different cohorts of COPD, a Swiss one to which the Living Well with COPD programme [9] was applied, with the International Collaborative Effort on Chronic Obstructive Lung Disease Exacerbation Risk Index Cohorts study [10] population as the control, the authors draw the conclusion that a coaching intervention based on self-management leading to empowerment and self-efficacy is feasible among COPD patients in a general practice setting, and provides a $64 \%$ rate reduction of acute exacerbation risk over time. In addition, health-related quality of life improved by a $\geqslant 0.54$-point reduction (on a seven-point scale) in each Chronic Respiratory Questionnaire domain score.

Therefore, it seems that the general practice in primary care settings represents a critical field of application for the CCM in order to reduce the impact on chronic respiratory patients in terms of both clinical burden and healthcare utilisation [11], even in the early phase of the disease. A major issue in this setting is thus represented by undiagnosed COPD patients, who would not benefit from the opportunity given by a CCM. Consequently, targeted case finding seems an essential step before strategies like health coaching and self-management support can be provided [12].

From the methodological point of view, a strength of the present study [8] is the comprehensive approach used, including information given to patients about the disease, coping skills and abilities, and self-confidence and proactive motivation for self-achievements. Indeed, each patient received group and individual one-on-one coaching sessions to assess and discuss all the physical and psychological aspects that might impact their resilience to the disease. All the interventions described and detailed in the study share a practical approach, and are focused on generating a tangible change in the patient's behavioural attitude to the disease. Moreover, the same authors provided a coherent cost-utility effect by following the effect of exacerbation rate over 2 years.

Despite the encouraging results, the lack of a randomised design with a control group within the same cohort still makes any generalisability unlikely. To attenuate the influence of this methodological limit on the entire analysis, the authors used a logistic regression model, namely the propensity score, to calculate the probability of each individual being in the treatment group. They completed the statistical correction by running a sensitivity analysis with controls restricted to those who may be offered a self-management intervention. Nevertheless, the use of the propensity scores and the sensitivity analysis cannot fully balance for the missing randomisation because of unpredictable confounding variables.

Notwithstanding, the findings of STEURER-STEY et al. [8], and the cost-saving impact in particular, warrant future studies to confirm. In the COPD population at large, the prevention of healthcare utilisation through a self-management approach to symptoms and care would heavily impact on the global disease burden.

With reference to this, the CCM needs to empower different aspects in order to gain effectiveness: 1) primary care-based constant follow-up of patients' improvement in self-efficacy; 2) tailored care processes on a per-patient basis; 3) achievement of integrated self-management abilities; 4) shared intervention by a multidisciplinary team of professionals; 5) adequate integration between the development of patient-oriented devices and any technical infrastructure; and 6) implementation of new technology in the self-management and self-efficacy process. With specific reference to the last two points, DE TOLEDO et al. [13] have reported that a telehealth care model, applied to 157 COPD patients, resulted in a reduction rate of re-admission as compared with controls (51\% and 33\%, respectively), with high acceptance amongst professionals, and low installation and exploitation costs. In a very recent report [14], detailed insights into the features of a patient-support mobile app integrated into a CCM delivered to COPD patients were presented. These include specific programmes for breathing techniques, stress and dyspnoea management, diet and nutrition and tools for personalised feedback, reminders, and social networking activities [15]. It could be argued that the integration of new technologies would represent the smarter and potentially greater improvement for the chronic care method, shifting the traditional approach towards a digital health for the CCM purposes.

\section{References}

1 Bodenheimer T, Wagner EH, Grumbach K. Improving primary care for patients with chronic illness: the Chronic Care Model, part 2. JAMA 2002; 288: 1909-1914.

2 Kaptein AA, Fischer MJ, Scharloo M. Self-management in patients with COPD: theoretical context, content, outcomes, and integration into clinical care. Int J Chron Obstruct Pulmon Dis 2014; 9: 907-917.

3 Australian Council on Safety and Quality in Health Care. Second national report on improving patient safety: improving medication safety. Canberra, Australian Council on Safety and Quality in Health Care, 2002.

4 Fromer L. Implementing chronic care for COPD: planned visits, care coordination, and patient empowerment for improved outcomes. Int J Chron Obstruct Pulmon Dis 2011; 6: 605-614. 
5 Effing T, Monninkhof EM, Van der Valk PD, et al. Self-management education for patients with chronic obstructive pulmonary disease. Cochrane Database Syst Rev 2007; 4: CD002990.

6 Erik WMA, Bischoff EWMA, Akkermans R, et al. Comprehensive self management and routine monitoring in chronic obstructive pulmonary disease patients in general practice: randomised controlled trial. BMJ 2012; 345 : e7642.

7 Benzo R, Vickers K, Novotny PJ, et al. Health coaching and chronic obstructive pulmonary disease rehospitalization. A randomized study. Am J Respir Crit Care Med 2016; 194: 672-680.

8 Steurer-Stey C, Dalla Lana K, Braun J, et al. Effects of the "Living well with COPD" intervention in primary care: a comparative study. Eur Respir J 2018; 51: 1701375.

9 Bourbeau J, Julien M, Maltais F, et al. Reduction of hospital utilization in patients with chronic obstructive pulmonary disease: a disease-specific self-management intervention. Arch Intern Med 2003; 163: 585-591.

10 Siebeling L, ter Riet G, van der Wal WM, et al. ICE COLD ERIC - International collaborative effort on chronic obstructive lung disease: exacerbation risk index cohorts - study protocol for an international COPD cohort study. BMC Pulm Med 2009; 9: 15.

11 Foot H, Freeman C, Hemming K, et al. Reducing Medical Admissions into Hospital through Optimising Medicines (REMAIN HOME) study: protocol for a stepped-wedge, cluster-randomised trial. BMJ Open 2017; 7 : e015301.

12 Jordan RE, Adab P, Sitch A, et al. Targeted case finding for chronic obstructive pulmonary disease versus routine practice in primary care (TargetCOPD): a cluster-randomised controlled trial. Lancet Respir Med 2016; 4: 720-730.

13 De Toledo P, Jiménez S, del Pozo F, et al. Telemedicine experience for chronic care in COPD. IEEE Trans Inf Technol Biomed 2006; 10: 567-573.

14 Sobnath DD, Philip N, Kayyali R, et al. Features of a mobile support app for patients with chronic obstructive pulmonary disease: literature review and current applications. JMIR Mhealth Uhealth 2017; 5: e17. 